\title{
Near-surface attenuation estimation using wave-propagation modeling
}

\author{
Nizare El Yadari ${ }^{1}$, Fabian Ernst ${ }^{2}$, and Wim Mulder ${ }^{1,2}$
}

\begin{abstract}
The effect of the near surface on seismic land data can be so severe that static corrections are insufficient. Full-waveform inversion followed by redatuming may be an alternative, but inversion will work only if the starting model is sufficiently close to the true model. As a first step toward determining a viscoelastic near-surface model, we assume that existing methods can provide a horizontally layered velocity and density model. Because near-surface attenuation is strongest, we propose a method to estimate the $\mathrm{P}$-wave attenuation based on viscoacoustic finite-difference modeling. We compare energy decay along traveltime curves of reflection and refraction events in the modeled and observed seismic data for a range of attenuation parameters. The best match provides an estimate of the attenuation. First, we estimate only the attenuation of the top layer and study the sensitivity to depth and velocity perturbations. Then, we consider multiple layers. We apply the method to synthetic and real data and investigate the effect of source wavelet and topography. The method is robust against depth and velocity perturbations smaller than $10 \%$. The results are sensitive to the source wavelet. Incorporating the surface topography in the computed traveltimes reduces the uncertainty of the attenuation estimates, especially for deeper layers.
\end{abstract}

\section{INTRODUCTION}

The near surface generates a variety of problems in land seismic data. The multiplicative effect resulting from statics and additive effects caused by guided waves, scattering, reverberations, ground roll, strong attenuation, heterogeneities, and topography mask and distort the deeper reflections. Filtering and static corrections (Booker et al., 1976; Robinson and Al-Husseini, 1982; Xia et al., 1999) are sometimes sufficient to clean up the data for further imaging. Where- as errors in short-wavelength statics reduce the signal-to-noise ratio $(\mathrm{S} / \mathrm{N})$ of the stack, errors in long-wavelength statics influence the structural image (Cox, 1999). This is especially a concern when the exploration target is a low-relief structure in an area with rough topography or complicated near surface. In some areas of the Middle East, long-wavelength statics may be $80-90 \mathrm{~ms}$ because of, for instance, sand dunes (Ley et al., 2003).

Predominantly, first arrivals are used to derive statics based on refraction tomography. Ernst (2007) shows that guided waves can constrain the long-wavelength part of the near-surface structure better than first arrivals. But these methods may fail for a complex near surface. Moreover, the precise definition of a reliable macromodel is still an open question - one that has only been addressed heuristically (Operto et al., 2000). An alternative is full-waveform inversion (Tarantola, 1984; Pratt, 1999; Pratt and Shipp 1999; Sirgue and Pratt, 2004; Pratt et al., 2005; Mulder and Plessix, 2006) followed by redatuming (Berryhill, 1979; Rogers, 1981; Berryhill, 1984; Shtivelman and Canning, 1988; Profeta et al., 1995; Mulder, 2005; Alkhalifah and Bagaini, 2006; Alkhalifah et al., 2007). Full-waveform inversion works only if the starting model is sufficiently close to the correct model.

Recent applications of frequency-domain full-waveform inversion provide detailed $\mathrm{P}$-wave velocity models for various geologic settings, ranging from overthrusts (Ravaut et al., 2004) through sedimentary basins (Malinowski and Operto, 2006) to subduction zones (Operto et al., 2006). These authors use the acoustic approximation to find the velocity model. However, the waveforms obviously contain much more information that might be used for multiparameter inversion - for instance, to determine density and attenuation. The most straightforward approach seems to be the viscoacoustic approximation (Ribodetti et al., 2007), under which joint inversion for velocity and attenuation might be performed.

Here we take a first step in determining an initial viscoacoustic near-surface model for data acquired in the Middle East. We assume that kinematic methods such as generalized linear inversion (GLI) (Cooke and Schneider, 1983), refraction statics (Cox, 1999), or guided waves (Ernst, 2007) provide the velocities and densities in a

Presented at the 77th Annual Meeting, SEG. Manuscript received by the Editor 26 June 2007; revised manuscript received 28 May 2008 ; published online 15 October 2008

${ }^{1}$ Delft University of Technology, Department of Geotechnology, Delft, The Netherlands. E-mail: n.elyadari@ tudelft.nl.

${ }^{2}$ Shell International Exploration and Production BV, Rijswijk, The Netherlands. E-mail: fabian.ernst@ shell.com; wim.mulder@shell.com. (C) 2008 Society of Exploration Geophysicists. All rights reserved. 
number of layers below the surface and try to estimate the attenuation parameters. Attenuation affects both amplitude and phase of all data, so we need accurate modeling. However, existing attenuationestimation methods (Hauge, 1981; Tonn, 1991) yield inaccurate results. A few approaches use the complete seismogram to find attenuation properties (Ribodetti and Virieux, 1998; Hicks and Pratt, 2001; Malinowski and Operto, 2007).

We present a method for estimating attenuation based on finitedifference viscoacoustic modeling, as outlined by El Yadari et al. (2007a, 2007b). Given velocities and densities in a layered medium, we compare the energy decay along traveltime curves in the modeled and observed seismic data for a range of attenuation parameters. The best match provides an estimate of the attenuation.

After a brief review of the theory of seismic attenuation, we describe the method. We define an error criterion that compares the energy of modeled and observed seismograms along traveltime curves. To validate the method, we consider synthetic data as well as real data. Our method involves frequency-dependent wave propagation for a layered model that may have a rough surface. Hence, we study the influence of the shape of the source wavelet and the effect of the local topography on the attenuation estimate, first for a single layer and then for multiple layers.

\section{THEORY OF SEISMIC ATTENUATION}

The literature on anelastic and rheological properties of earth materials is vast. As a starting point for the interested reader, we mention a book by Christensen (1982) on viscoelasticity in general and a geophysically oriented review by Minster (1980).

Dissipation or absorption of wave energy is often described by linear laws, i.e., we assume that stress and strain are related linearly as in purely elastic media. The difference from viscoelastic media is that phase shifts now occur between stress $\sigma$ and strain $\varepsilon$. This implies that the elastic moduli are no longer real but complex and possibly frequency dependent. The simple 1D stress-strain relation is

$$
\sigma(\omega)=M(\omega) \varepsilon(\omega)
$$

We call $M(\omega)$ the viscoelastic modulus without specifying the mode of deformation. The viscoelastic modulus is separated into real and imaginary parts, $M=M_{1}+i M_{2}$, or into magnitude $A$ and phase $\varphi$, $M=A e^{i \varphi}$, where $i$ is the imaginary unit. All these quantities, in principle, depend on frequency. The most common measure of attenuation is the dimensionless quality factor $Q$, defined by

$$
Q^{-1}=\frac{M_{2}}{M_{1}}=\tan (\varphi)
$$

Increasing dissipation increases the phase shift $\varphi$ between stress and strain and hence decreases $Q$. We can show that if $Q \gg 1, Q^{-1}$ is proportional to the energy loss per period in a harmonic-loading experiment and therefore has a simple physical meaning, $Q$ can be measured by different techniques. We refer to Blanch et al. (1998), Dasgupta and Clark (1998), Ayres and Theilen (2001), and Xia et al. (2002), including amplitude measurements of propagating waves, width measurements of spectral lines in spectra of free oscillations (Rickett, 2006, 2007), and, of course, phase-shift measurements between stress and strain in forced oscillations.
An important point is that equation 1 can be considered a linear filter. The filter, represented by the viscoelastic modulus, must be causal, i.e., the filtered output $\sigma(t)$ in the time domain must not start earlier than the input $\varepsilon(t)$. This requirement imposes relations between $M_{1}$ and $M_{2}$, or $A$ and $\varphi$, which are called dispersion or Kramers-Kronig relations. Those relating magnitude $A$ and phase $\varphi$ (and hence $Q$, according to equation 2) are the most important in the present context:

$$
\begin{gathered}
\ln A(\omega)=B-\frac{1}{\pi} P \int_{-\infty}^{+\infty} \frac{\varphi(\omega)}{\omega^{\prime}-\omega} d \omega^{\prime}, \\
\varphi(\omega)=\frac{1}{\pi} P \int_{-\infty}^{+\infty} \frac{\ln A(\omega)}{\omega^{\prime}-\omega} d \omega^{\prime},
\end{gathered}
$$

where $P$ is the principal-value integral.

For simple types of frequency dependence of $Q$, the principal-value integral can be calculated analytically and the constant $B$ can be determined either at high or low frequencies. As a consequence, the viscoelastic modulus is known for all frequencies. If this procedure is followed for the rigidity $\mu(\omega)$ and the bulk modulus $k(\omega)$, and if these complex moduli are used instead of the real moduli to solve an elastic wave-propagation problem, then the frequency-domain solution of the corresponding viscoelastic problem is obtained. This is the correspondence principle of the linear theory of viscoelasticity.

Working with $\mu(\omega)$ and $k(\omega)$ is, however, not the normal procedure. Usually, we work with wave velocities and hence replace real velocities by complex velocities. For $\mathrm{P}$ - and $\mathrm{S}$-waves, we have the complex velocities

$$
V_{\mathrm{P}}(\omega)=\left[\frac{M_{\mathrm{P}}(\omega)}{\rho}\right], \quad V_{\mathrm{S}}(\omega)=\left[\frac{M_{\mathrm{S}}(\omega)}{\rho}\right]^{1 / 2} .
$$

Here, $\rho$ is the real density. The viscoelastic moduli for P- and $\mathrm{S}$-waves are

$$
M_{\mathrm{P}}(\omega)=k(\omega)+\frac{4}{3} \mu(\omega), \quad M_{\mathrm{S}}(\omega)=\mu(\omega),
$$

with the quality factors $Q_{\mathrm{P}}$ and $Q_{\mathrm{S}}$ following from

$$
Q_{\mathrm{P}}^{-1}=\frac{4 v_{\mathrm{P}}^{2}}{3 v_{\mathrm{S}}^{2}} Q_{\mu}^{-1}+\left(1-\frac{4 v_{\mathrm{P}}^{2}}{3 v_{\mathrm{S}}^{2}}\right) Q_{k}^{-1}, Q_{\mathrm{S}}=Q_{\mathrm{n}} .
$$

Here, $Q_{\mu}$ and $Q_{k}$ are the quality factors of $\mu$ and $k$, respectively, and $v_{\mathrm{P}}$ and $v_{\mathrm{S}}$ are real wave velocities at a typical frequency. A familiar assumption is that $Q_{k} \gg Q_{\mu}$, i.e., there is much less dissipation in volume deformation than in shear deformation. Then $Q_{\mathrm{P}}$ depends only on $Q_{\mu}$, and $Q_{\mathrm{P}}$ and $Q_{\mathrm{S}}$ have the same frequency dependence. This implies a real frequency-independent bulk modulus at least in the seismic-frequency band. An often-used relation is $Q_{\mathrm{P}}=2.25 Q_{\mathrm{S}}$, based on $v_{\mathrm{P}}^{2}=3 v_{\mathrm{S}}^{2}$.

The procedure to find complex velocities is to make assumptions about $Q_{\mathrm{P}}$ and $Q_{\mathrm{S}}$ as functions of frequency, to use equation 5 and 6 to determine $M_{\mathrm{P}}$ and $M_{\mathrm{S}}$, and then to insert these moduli into equation 
4. In the following, we disregard the distinction of P- and S-waves and work with $M(\omega), Q(\omega)$, and the complex velocity:

$$
V(\omega)=\left[\frac{M(\omega)}{\rho}\right]^{1 / 2}
$$

If $Q$ is a frequency-independent constant or if it follows from the power law that

$$
Q(\omega)=Q\left(\omega_{r}\right)\left[\frac{\omega}{\omega_{r}}\right]^{\gamma}
$$

with reference frequency $\omega_{r}$ and an exponent $\gamma$ between -1 and +1 , the steps leading to the complex velocity are rather straightforward (Muller, 1983).

These $Q$ laws and related absorption-band models have been investigated many times in the literature (see Minster, 1980). Here we consider only the case of seismologic interest, where $Q$ is a frequency-independent constant and $Q \gg 1$. In the case of a layered medium, we include the attenuation by adding an imaginary part to the velocity $V_{m}$ for each layer $(m=1$ to $M$ ). The modified velocity in the $n$th layer is

$$
\hat{V}_{m}=V\left(1-\frac{i}{2 Q_{r}}\right)
$$

Consequently, for $\left(1 / 2 Q_{r}\right) \ll 1$, attenuation causes the wave amplitude to be multiplied by $e^{-\delta x}$, where $\delta=\omega /\left(2 V Q_{r}\right)$ and $x$ is the distance in the direction of propagation.

In addition, causal absorption involves an extra frequency-dependent term modifying the real part of the velocity. The effects of this term and references to its introduction by Lomnitz are discussed in O'Neil and Hill (1979). We use an extra term, which results in the expression

$$
\hat{V}_{m}=V\left(1-\frac{i}{2 Q_{r}}-\frac{1}{\pi Q_{r}} \ln \left(\frac{\omega}{\omega_{r}}\right)\right) .
$$

In the case of the power law,

$\hat{V}_{m}=V\left\{1-\frac{1}{2}\left[\frac{1}{Q\left(\omega_{r}\right)}-\frac{1}{Q(\omega)}\right] \cot \left(\frac{\gamma^{\pi}}{2}\right)-\frac{i}{2 Q(\omega)}\right\}$.

O'Neil and Hill (1979) discuss the selection of the reference frequency $\omega_{r}$ and the modified quality factor $Q_{r}$.

An alternative to the use of complex velocities is the application of dissipation operators that are convolved with seismograms computed for purely elastic media (Carcione et al., 1988; Blanch et al., 1995). Dissipation operators follow from the plane-wave delta-function response of a homogeneous absorbing medium by appropriately averaging the $Q$-factor of an inhomogeneous medium along seismic rays. The use of such operators, usually corresponding to a frequency-independent $Q$, is common in seismogram calculations by generalized or asymptotic ray theory. Operators for the power law (equation 11) are given by Muller (1983). The use of dissipation operators, however, is restricted to body-wave investigations with little or no interference of phases propagating along different rays. Interfering body waves and surface waves usually must be treated with the aid of complex velocities.

\section{METHOD}

From an observed seismogram, we want to determine a good initial viscoelastic near-surface model that can be used for full-waveform tomography. Here, we make a first step by only considering the simpler viscoacoustic case and concentrating on $\mathrm{P}$-wave reflections and refractions. To find a starting model, kinematic methodic refraction statics (e.g., Coppens, 1985; Zanzi, 1990; Zanzi and Carlini, 1991; Cox, 1999; Bohm et al., 2006) can provide a horizontally layered model with density $\rho_{m}$, depth $d_{m}$, and velocity $V_{m}$ for each layer. In such a model, we try to estimate the attenuation in the near surface where it is strongest. Our method may also be suited for lateral-velocity variations and rough reflectors. Here, we assume that $\boldsymbol{\rho}, \mathbf{d}$, and $\mathbf{V}$ (vectors with a length $M$ equal to the number of layers) are known, and we only want to estimate the attenuation parameters, denoted by the vector $\mathbf{Q}$. Figure 1 shows the various steps of the method.

The subsurface model is assumed to be a layered medium with $Q_{m}$ constant in each layer. A sequence of $N$ models $\left\{\mathbf{Q}^{1}, \mathbf{Q}^{2}, \ldots, \mathbf{Q}^{N}\right\}$, characterized by $\mathbf{Q}^{n}=\left(Q_{1}^{n}, Q_{2}^{n}, \ldots, Q_{M}^{n}\right)^{T}, n=1$ to $N$, is derived on a regular grid of values by starting from an initial guess $\mathbf{Q}^{0}$. The latter can be obtained arbitrarily or by using, for example, another attenuation estimation method (Toksöz and Johnston, 1981). Among all of these $N$ models, we want to find the most likely one. We use a $2 \mathrm{D}$ viscoacoustic, finite-difference, frequency-domain modeling code to simulate the full wavefield for each $n$th subsurface model, denoted by $\mathbf{Q}^{n}$. The modeled seismogram resulting from each simulation is then compared with what we call the observed seismogram.

In the following, the observed seismogram can be either real or synthetic. In this way, we can find the most likely attenuation model, denoted by $\mathbf{Q}^{\text {est }}$. We do not compare the full seismograms, as in fullwaveform inversion, but only consider amplitudes along traveltime curves for refracted and reflected waves. This circumvents some of the problems related to the viscoacoustic approximation and the illposed character of the inverse problem. We pick these curves from each synthetic and observed seismogram and select the corresponding amplitudes, denoted by $A^{\text {synth }}$ and $A^{\text {obs }}$, respectively. The mini-

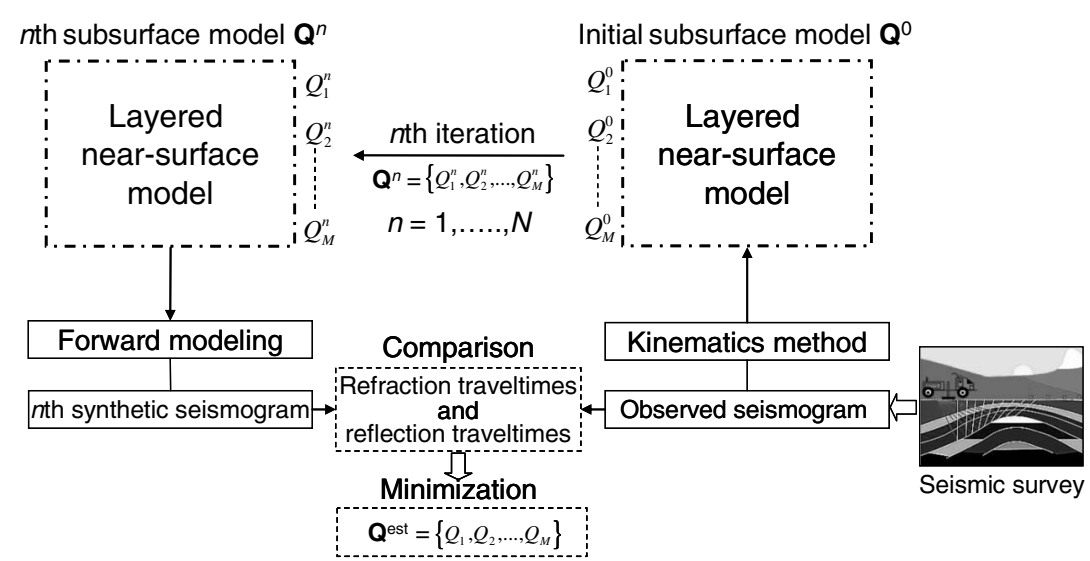

Figure 1. Overview of the method for estimating attenuation using wave-propagation modeling. 
mum value of a specific error criterion determines the most likely $\mathbf{Q}^{\text {est }}$.

\section{Error criterion}

We define an error criterion that measures the difference between the observed and synthetic seismograms as

$$
E\left(\mathbf{Q}^{n}\right)=\sum_{m=1}^{M} \alpha_{m} E_{m}\left(\mathbf{Q}^{n}\right),
$$

with $\alpha_{m}$ the weight assigned to the $m$ th layer. We impose

$$
\sum_{m=1}^{M} \alpha_{m}=1
$$

In equation 12, $E_{m}\left(\mathbf{Q}^{n}\right)$ is the error corresponding to the $m$ th layer, calculated by

$$
E_{m}\left(\mathbf{Q}^{n}\right)=E_{m}^{\mathrm{refl}}\left(\mathbf{Q}^{n}\right)+E_{m}^{\mathrm{refr}}\left(\mathbf{Q}^{n}\right)
$$

where $E_{m}^{\text {refl }}$ and $E_{m}^{\text {refr }}$ are the errors for reflected and refracted events, respectively. As a first step, $E_{m}^{\text {refl/refr }}\left(\mathbf{Q}^{n}\right)$ can be defined as

$E_{m}^{\mathrm{refl} / \mathrm{refr}}\left(\mathbf{Q}^{n}\right)=\sum_{t=\text { traces }}\left|\log \left(\frac{A^{\mathrm{obs}}\left(T_{m}^{\mathrm{refl} / \mathrm{refr}}\left(H_{t}\right)\right)}{A_{n}^{\mathrm{syn}}\left(T_{m}^{\mathrm{refl} / \mathrm{refr}}\left(H_{t}\right)\right)}\right)\right|$,

if we measure the difference between the picked amplitudes. Note that $T_{m}^{\mathrm{refl}}\left(H_{t}\right)$ and $T_{m}^{\mathrm{refr}}\left(H_{t}\right)$ correspond, respectively, to the traveltime of a ray reflected or refracted along the top surface of the $m$ th layer for a source-receiver distance, denoted by $H_{t} . A^{\text {syn }}$ and $A^{\text {obs }}$ are the picked amplitudes from the synthetic and observed seismograms, respectively.

Above, we mention that the wave amplitude is proportional to $e^{-\beta / Q}$, where $\beta=\omega x /(2 V)$. Consequently, the use of the logarithm will allow us to measure $\mathbf{Q}$ directly. However, the use of amplitude misfits at a single point in time seems risky, especially for problems including many unknowns, as is the case for real data. Uncertainties in the flatness of the reflectors, their depth, the velocity model, unidentified heterogeneities, and lateral variations may introduce additional phase shifts and modify the general shape of the observed signal. Therefore, a discrete point-error value is likely to be affected by all such factors.

As an alternative, we measure the difference between the energy inside a window, denoted by $W_{t}$, around $A^{\text {syn }}$ and $A^{\text {obs }}$. We denote these differences by $\varepsilon^{\text {syn }}$ and $\varepsilon^{\text {obs }}$, respectively. Following Max (1972), we define the scalar product of two analytic signals $\widetilde{x}(t)$ and $\tilde{y}(t)$ around the window $W_{t}$ as

$$
<\tilde{x}, \tilde{y}>_{W_{t}}=\int_{W_{t} / 2}^{W_{t} / 2} \tilde{x}(t) \overline{\tilde{y}(t)} d t,
$$

where $\widetilde{x}(t)=x(t)+i H(x)(t)$ with $H(x)$ is the Hilbert transform of $x(t)$. Hence, we express the energy $\varepsilon$ inside the window $w_{t}$ as

$$
\varepsilon=<\tilde{\mathrm{A}}, \tilde{\mathrm{A}}>_{W_{t}}
$$

Then

$$
E_{m}^{\mathrm{refl} / \mathrm{refr}}\left(\mathbf{Q}^{n}, W_{t}\right)=\sum_{t=\text { traces }}\left|\log \left(\frac{\varepsilon^{\mathrm{obs}}\left(T_{m}^{\mathrm{refl} / \mathrm{refr}}\left(H_{t}\right)\right)}{\varepsilon_{n}^{\mathrm{syn}}\left(T_{m}^{\mathrm{refl} / \mathrm{refr}}\left(H_{t}\right)\right)}\right)\right| .
$$

\section{Picking traveltimes}

Traveltime picking consists of finding the traveltime corresponding to the peak (maximum amplitude) closest to the one calculated by the following formulas. The traveltime of a ray refracted along the top flat surface of the $m$ th layer for a source-receiver distance, denoted by $H$, is given by

$$
t_{m}^{\mathrm{refr}}(H)=\frac{H}{V_{m}}+2 \sum_{j=1}^{m-1} d_{m}\left(\frac{1}{V_{j}^{2}}-\frac{1}{V_{m}^{2}}\right)^{1 / 2} .
$$

If the ray is reflected, the traveltime expression will be of the form

$$
t_{m}^{\mathrm{refl}}(H)=\sqrt{T_{\mathrm{tot}}^{2}(0)+\frac{H^{2}}{V_{\mathrm{rms}}^{2}}},
$$

with $V_{\mathrm{rms}}$ the root-mean-square velocity defined by

$$
V_{\mathrm{rms}}^{2}=\frac{1}{T_{\mathrm{tot}}(0)} \sum_{j=1}^{m} V_{j}^{2} T_{j}(0)
$$

where $T_{j}(0)=2 d_{j} / V_{j}$ denotes the zero-offset traveltime through the $j$ th layer with a thickness of $d_{j}$ and $T_{\text {tot }}(0)$ denotes the total zero-offset time:

$$
T_{\mathrm{tot}}(0)=\sum_{j=1}^{m} T_{j}(0)
$$

Because of the presence of rough topography of the local surface, we must include elevation corrections when calculating these traveltimes (see Figure 2). Then
Figure 2. Schematic of a ray reflected or refracted along the top surface of a layer.

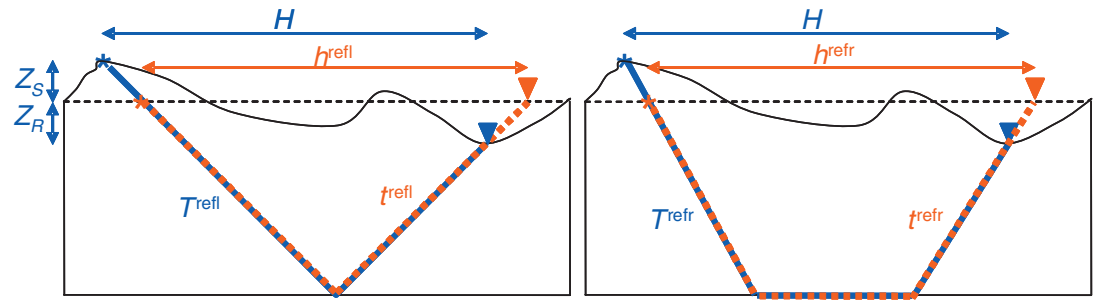




$$
T_{m}^{\mathrm{refr} / \mathrm{refl}}(H)=t_{m}^{\mathrm{refr} / \mathrm{refl}}\left(h^{\mathrm{refr} / \mathrm{refl}}\right)-\Delta t^{\mathrm{refr} / \mathrm{refl}},
$$

with

$$
h^{\mathrm{refr}}=H+\frac{z_{r}+z_{s}}{\sqrt{\left(\frac{V_{2}}{V_{1}}\right)^{2}-1}} \text { and } h^{\mathrm{refl}}=\frac{H}{1-\frac{z_{r}+z_{s}}{2 d_{1}}} .
$$

Here $V_{1}$ and $V_{2}$ are the velocities of the first and second layers, and $d_{1}$ is the thickness of the first layer. These quantities are assumed constant. The terms $z_{s}$ and $z_{r}$ denote the source and the receiver elevation corrections to $d_{1}$, respectively. The elevation corrections for refracted and reflected traveltimes are given by

$$
\Delta t^{\mathrm{refr}}=\frac{z_{s}+z_{r}}{V_{1}} \frac{1}{\sqrt{1-\left(\frac{V_{1}}{V_{2}}\right)^{2}}}
$$

and

$$
\Delta t^{\mathrm{refl}}=\frac{z_{s}+z_{r}}{V_{1}} \sqrt{1+\left(\frac{h^{\mathrm{refl}}}{2 d_{1}}\right)^{2}} .
$$

\section{SYNTHETIC RESULTS}

Before applying our method to real data, we start with a number of synthetic tests. These tests amount to an inverse crime but allow us to validate our method and analyze various effects. To achieve this, we first describe the forward-modeling part. Then, we treat the effect of different wave paths on the error. Finally, we examine the influence of the shape of the source wavelet on the attenuation estimate.

\section{Forward modeling}

To generate the amplitudes for the modeled seismograms, we must use forward modeling. The forward modeling employs the finite-difference scheme described in Mulder and Plessix (2004). We only considered 2D problems. We want to determine an initial near-surface model for data acquired in the Middle East, for which a subsurface model with the features illustrated in Figure $3 \mathrm{a}$ was available from refraction statics. The first layer reaches a depth of $50 \mathrm{~m}$; the second, $110 \mathrm{~m}$; the third, $360 \mathrm{~m}$; and the fourth, $500 \mathrm{~m}$. The receivers are located between 63 and $2963 \mathrm{~m}$ at a spacing of $25 \mathrm{~m}$ on one side of an impulsive source at 3-m depth. For the modeling, we used frequencies between 8 and $20 \mathrm{~Hz}$ based on the source spectrum. The domain size was $3100 \times 500 \mathrm{~m}$. The uniform grid distance of the rectangular numerical grid size was $5 \mathrm{~m}$ and was discretized by $620 \times 100$ grid points.

The acoustic pressure was set to zero at the free surface, whereas the other boundaries were ab- sorbing. For this, we used the second-order Engquist-Majda (1977) boundary condition augmented by an extra boundary strip with increasing $Q$. To transform the synthetic seismogram computed with the frequency-domain code to the time domain, we used one of the two source wavelets displayed in Figure 4. Note that the wavelet at the left side is estimated from the real data, whereas the other one is chosen wrong intentionally.

\section{Effect of different wave paths on the error}

Our method uses weight parameters $\alpha_{m}$. Also, we measure the energy in a strip around the picked traveltime curve of temporal width $W_{t}$. Consequently, we have to choose the particular values of $\alpha_{m}$ and $W_{t}$. Figure 5 presents a study of the relationship between these parameters for pairs of $Q_{m}$. To describe this, we only allow $Q_{1}$ and $Q_{2}$ to vary and assume fixed $Q_{3}$ and $Q_{4}$ equal to 100 . We computed 441 synthetic seismograms for $Q_{1}=Q_{2}=4,6, \ldots, 44$, using the wavelet $a$ in Figure 4. These seismograms were then compared successively with the one corresponding to $Q_{1}$ and $Q_{2}$ equal to 24 .

In the top row of Figure 5, we show the curves corresponding to refraction and reflection traveltimes picked from the observed seismogram and calculated separately for the first, second, and third layers. The last column shows all of them. To evaluate the effect of the window size on the obtained energy, we calculated $\varepsilon$ for four different values of $W_{t}$ equal to $0.05,0.1,0.2$, and $1 \mathrm{~s}$. These values correspond to $0.5,1,2$, and 10 wavelengths, respectively. In the second row of Figure 5, we display the curves representing the amplitude decay along refracted and reflected traveltime curves for each layer and their sum. In the third row, we present the curves of their corre-
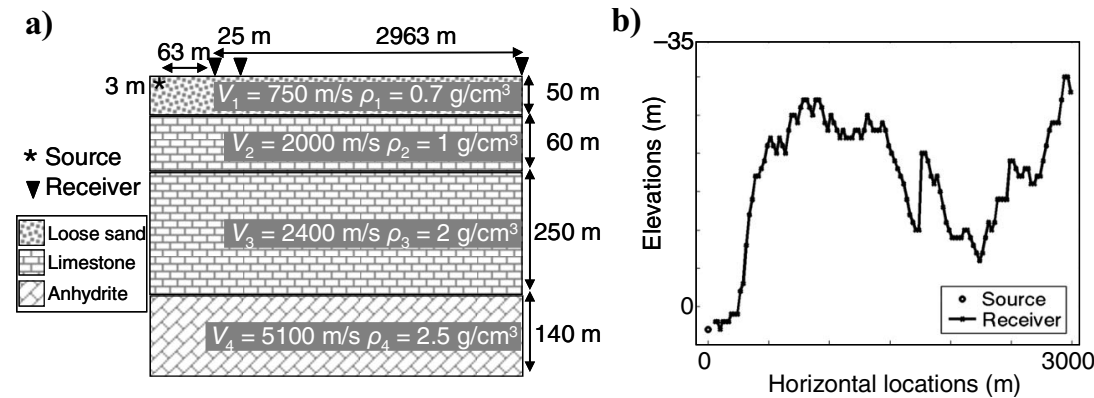

Figure 3. (a) Subsurface model obtained from refraction statics. (b) Elevations of the source and receivers. a)
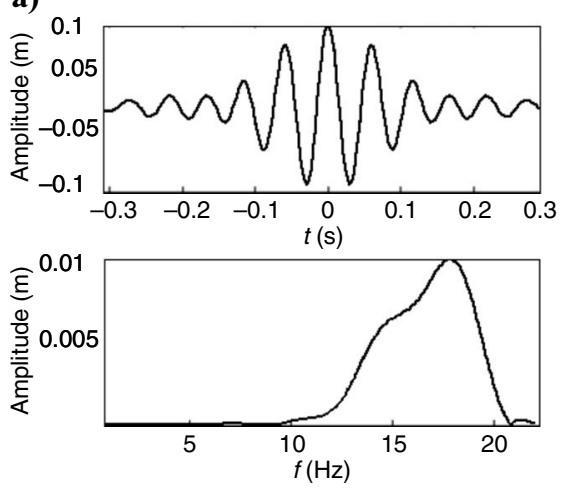

b)
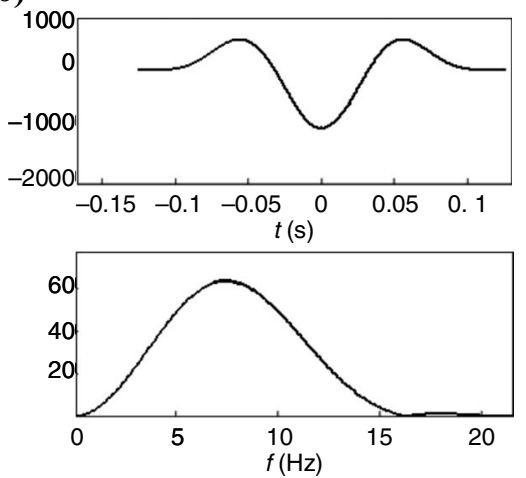

Figure 4. Estimated wavelets and their corresponding spectra used to convert the seismogram to the time domain. (a) From real data. (b) Intentionally chosen wrong. 
sponding energy decay inserted into the error criterion. At the bottom of Figure 5, we only display the contributions of the error for $W_{t}$ $=0.1 \mathrm{~s}$ as calculated for each layer, $E_{1}, E_{2}$, and $E_{3}$, and their total sum $E$. This corresponds to $\alpha_{1}, \alpha_{2}$, or $\alpha_{3}=1$ (with the other $\alpha_{m}$ equal to zero), whereas the sum $E$ was obtained with $\alpha_{1}=\alpha_{2}=\alpha_{3}$ $=1 / 3$.

The bottom row of Figure 5 displays the error surfaces for each layer and, in the fourth column, for their sum. Ideally, these error surfaces should have a well-defined minimum at the correct value of the attenuation parameters, meaning that the error image should be bowl shaped with blue at the correct minimum and rapidly, smoothly increasing values, from green to red, away from the minimum. The sharper the minimum, the better the resolution. In Figure 5, we observe that the minimum becomes less well defined if we go from the first to the third layer, implying that the resolution deteriorates if we go to the deeper layers. We might have considered the use of larger values of $\alpha_{m}$ for those layers that provide a better resolution but did not do so.
Next, we investigate the effect of the window width $W_{t}$. Figure 6 shows that the error images obtained for $\alpha_{1}=\alpha_{2}=\alpha_{3}=1 / 3$ are quite similar for $W_{t}=0.05,0.1$, and $0.2 \mathrm{~s}$. These can be considered reasonable values based on their associated wavelength. For the fourth case, $W_{t}=1 \mathrm{~s}$, the resolution of the error image deteriorates. Note the reasonably smooth behavior of all error images. Also, the correct parameter is always obtained at the minimum indicated by the dashed lines. Furthermore, one set of parameters we use to validate how well our error criterion can solve the comparison between the modeled and the observed seismograms is $\alpha_{1}=\alpha_{2}=\alpha_{3}=1 / 3$ and $W_{t}=0.1 \mathrm{~s}$.

We justify our choice of alpha values by the intrinsic behavior of the attenuation in upper and deeper layers: $Q_{1}$ may affect $Q_{2}, Q_{3}$, and $Q_{4}$. Also, $Q_{1}$ and $Q_{2}$ may affect $Q_{3}$ and $Q_{4}$, and so on. For this reason, we prefer to work with $\alpha_{1}=\alpha_{2}=\alpha_{3}=1 / 3$. Note that $\alpha_{4}$ is equal to zero because the bottom of the fourth layer is absorbing and does not generate reflection or refraction events.

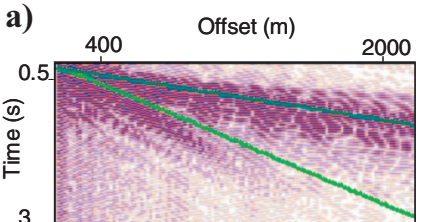

b)
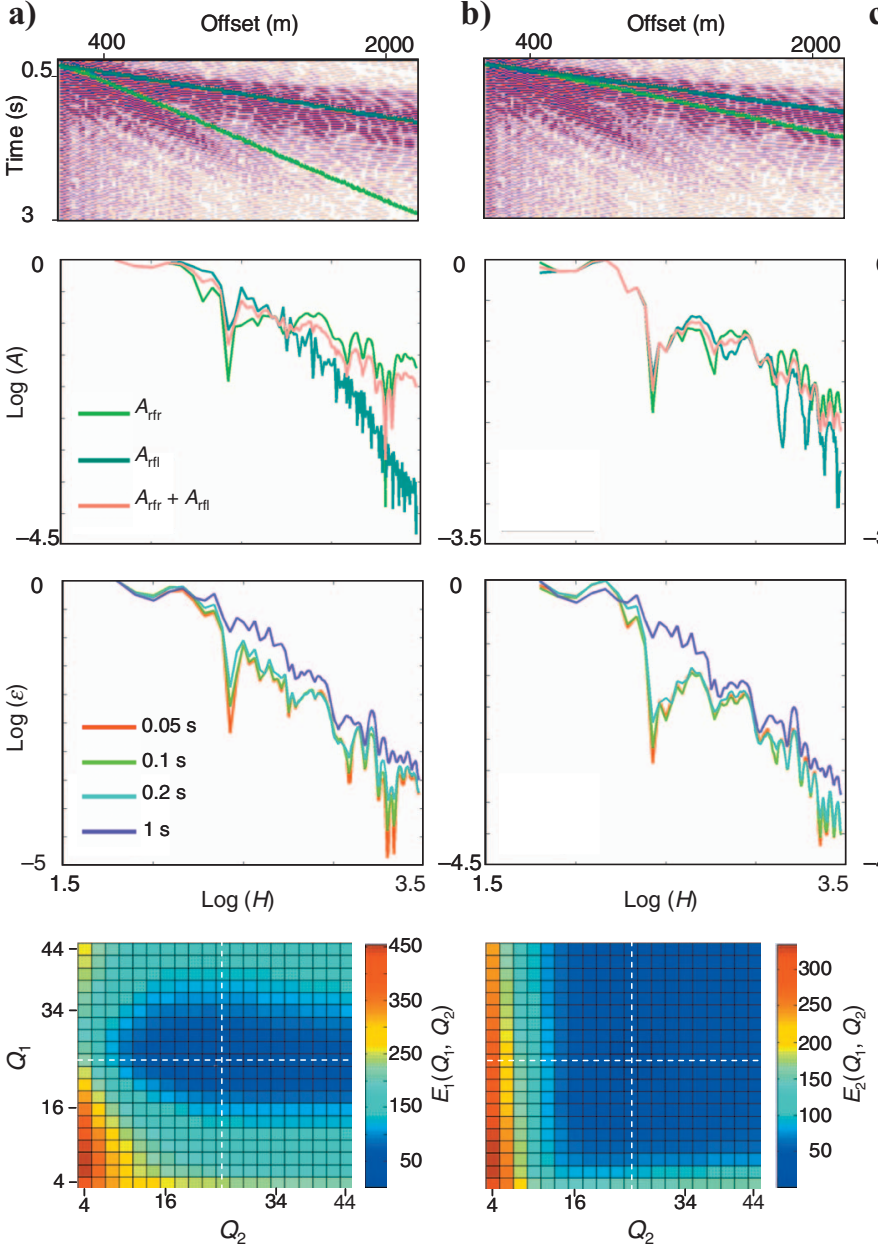
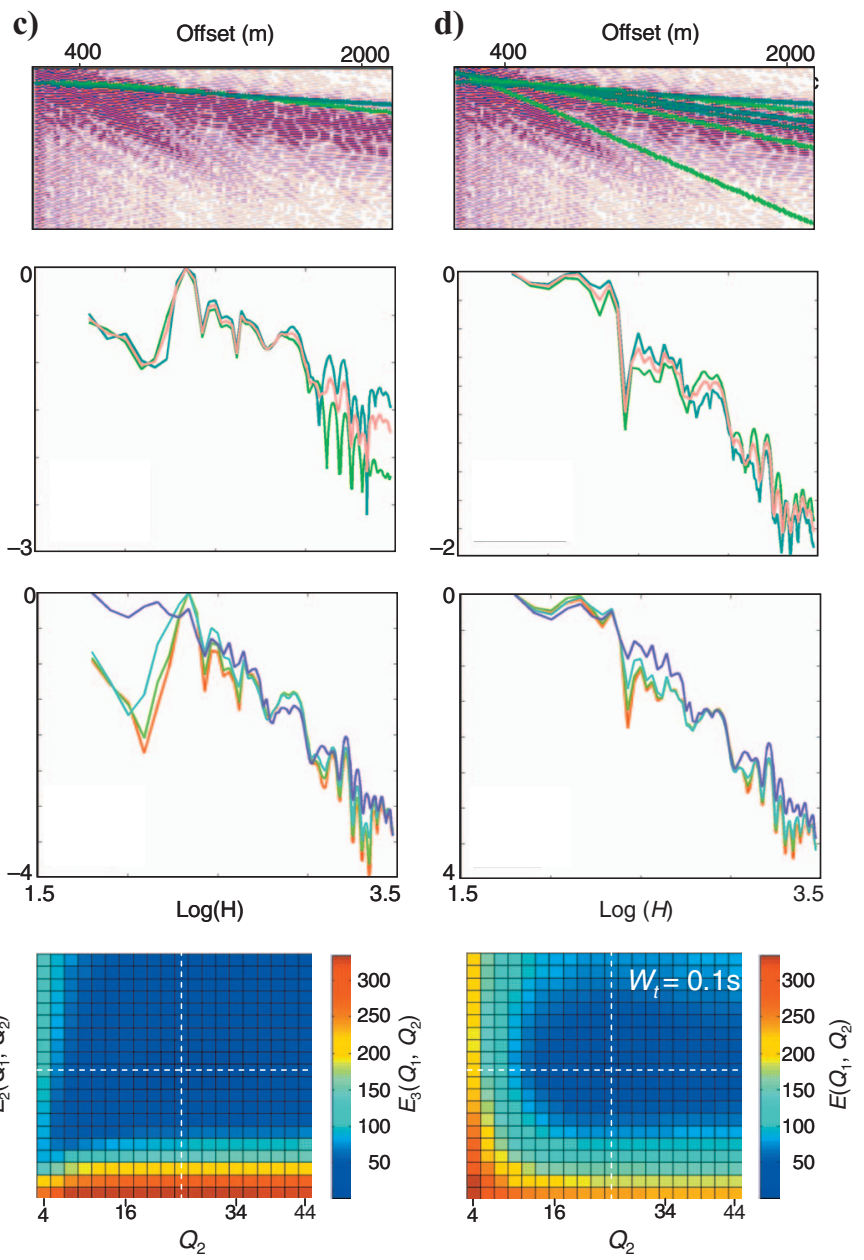

Figure 5. (Row 1) Refraction (blue line) and reflection (green line) traveltime curves picked on traces from the observed seismogram and calculated separately for the (a) first, (b) second, and (c) third layers as well as (d) their total sum. (Row 2) Amplitude decays along the refracted and reflected traveltime curves and their sum (red line). (Row 3 ) Corresponding energy decays for several values of window size $W_{t}$. (Row 4$)$ Error surfaces as a function of $Q_{1}$ and $Q_{2}$ for fixed $Q_{3}$ and $Q_{4}$, calculated only with $W_{t}=0.1 \mathrm{~s}$, shown separately for (a) the first layer, $E_{1}\left(Q_{1}, Q_{2}\right) ;(\mathrm{b})$ the second layer, $E_{2}\left(Q_{1}, Q_{2}\right)$; (c) the third layer, $E_{3}\left(Q_{1}, Q_{2}\right)$; and (d) their total sum, $E\left(Q_{1}, Q_{2}\right)$. The white dashed lines indicate the position of the minimum. 


\section{Influence of the shape of the source wavelet}

Because our method involves picking amplitudes from the complete seismogram, the spectrum of the source wavelet is important. We therefore studied the influence of the shape of the estimated wavelet. We used the two different estimated wavelets shown in Figure 4 to transform the synthetic seismogram computed with the frequency-domain code to the time domain. We utilized the same 441 synthetic seismograms as in the previous section. To evaluate the effect of the inaccuracy of the estimated wavelet, the 441 seismograms obtained with wavelet $a$ were compared to the observed seismogram calculated with $Q_{1}$ and $\mathrm{Q}_{2}$ each equal 24 and transformed to the time domain, either with the wavelet in Figure 4a or with the wavelet in Figure 4b.

Figure 7 shows the resulting error images. Figure 7 a corresponds to using the correct wavelet; Figure $7 \mathrm{~b}$ corresponds to the wrong wavelet. The comparison shows how strong the effect of the estimated wavelet can be. This can be explained easily by realizing how traveltimes were picked. We pick the maximum-amplitude peak nearest the one predicted by the traveltime formulas. We consider the energy inside a window around the considered peak. Consequently, a change in the shape of the wavelet will affect the picked amplitude and the resulting values of the error.

\section{FIELD DATA}

We applied our method to a 2D data set recorded in the Middle East. There, part of the energy is converted from $\mathrm{P}$ to $\mathrm{S}$ modes, reducing the total amplitude of some picked refracted or reflected events. Also, Rayleigh and guided waves are strong. Futhermore, wave propagation for real data occurs in a 3D medium. We applied a rather crude 2D-to-3D correction (Crase et al., 1990). Consequently, we expect that our 2D viscoacoustic modeling will not provide the correct value of $\mathbf{Q}^{\text {est }}$. Nevertheless, we want to verify that our method provides a well-defined result before we enter into the complexities of the viscoelastic case.
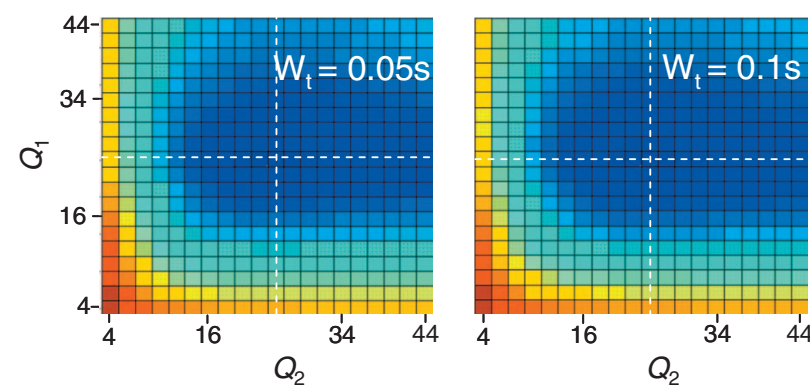

The application to synthetic data demonstrates the need for a correct estimate of the wavelet. To determine the wavelet for the field data, a near-offset trace was zero phased. We only kept the part of its spectrum between 8 and $20 \mathrm{~Hz}$. The resulting wavelet in Figure 4a.

To construct an attenuation model, we start with processing in a layer-stripping fashion to estimate the attenuation $Q_{1}$ in the top layer. For simplicity, we only study the sensitivity of the technique to the depth of the reflectors and the velocity model. Next, we extend the method to multiple layers and examine the effect of the local topography on the attenuation estimate. Once $Q_{1}$ is determined, we try to estimate $Q_{2}$, and so on, up to $Q_{3}$.

\section{Estimating $Q_{1}$}

To estimate $Q_{1}$, we constructed 21 synthetic seismograms for constant values of $Q_{2}, Q_{3}$, and $Q_{4}=100$ but for $Q_{1}=4,6, \ldots, 44$. Here, we are only interested in the first layer. Therefore, we assume a high value for $Q_{2}, Q_{3}$, and $Q_{4}$ equal to 100 . This assumption is equivalent to considering only weak attenuation in those layers. Of course, the estimated $Q_{1}$ will not be accurate because refracted waves also occur in the second layer. For this reason, we need to incorporate at least one deeper layer (i.e., $Q_{2}$ ); this is studied in the next section.

The 21 modeled seismograms are compared successively with the observed one. By analogy with Figure 5, we show from the top to the bottom of Figure 8 - separately for the first, second, and third layers and their combination - traveltimes curves picked from the real data and their corresponding amplitude decay, as well as the energy decay for the same values of $W_{t}$ as in the above for the synthetic test. The values of the energy calculated for $W_{t}=0.1 \mathrm{~s}$ are then inserted into the error criterion to estimate how well $Q_{1}$ explains the data. Note that these values are calculated for each layer and are normalized by their maximum over each corresponding layer. However, the energy decay exhibit jumps and their corresponding traveltime curves do not always correspond to the correct event. This may affect the attenuation estimate.
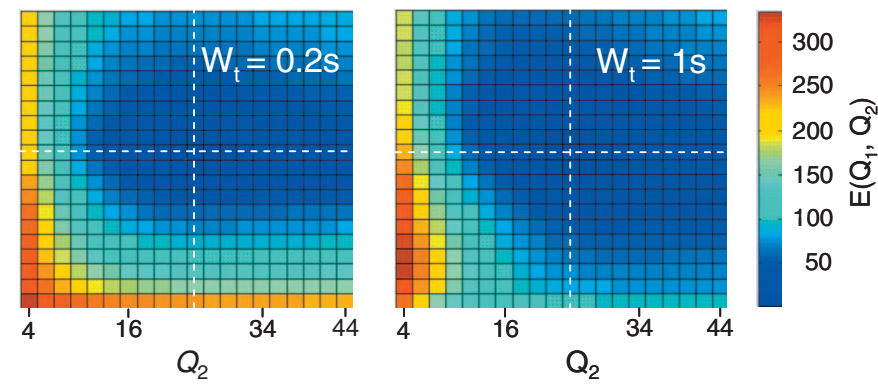

Figure 6. Error surfaces as a function of $Q_{1}$ and $Q_{2}$ for fixed $Q_{3}$ and $Q_{4}$, calculated with $\alpha_{1}=\alpha_{2}=\alpha_{3}=1 / 3$ for four window sizes, denoted by $W_{t}$.
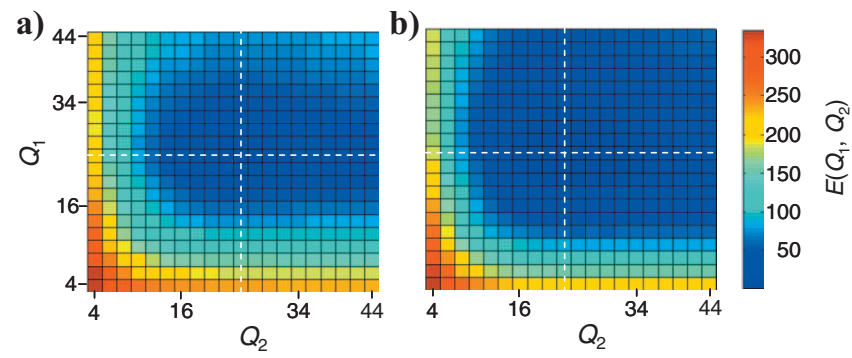

Figure 7. Error images, showing the influence of the estimated wavelet. (a) Use of the correct wavelet. (b) Use of the wrong wavelet. 
To evaluate the sensitivity of the error criterion, we performed a robustness study by perturbing separately and simultaneously the depth of the interfaces and the velocity model. Figure 9 presents results obtained with repeating simulation and only perturbing the first layer from its initial value. For this, we computed three new sets of 21 synthetic seismograms with perturbations up to $+10 \%$ of either the velocity or depth, or both. Figure 9 shows the error curves are quite similar. Also, their minima are located around the same value of $Q_{1}$. Note the reasonably smooth behavior of the curves. The minimum value for the real data occurs at $Q_{1}=24$. This means our attenuation method is robust against perturbations of depth and velocity of the first layer.
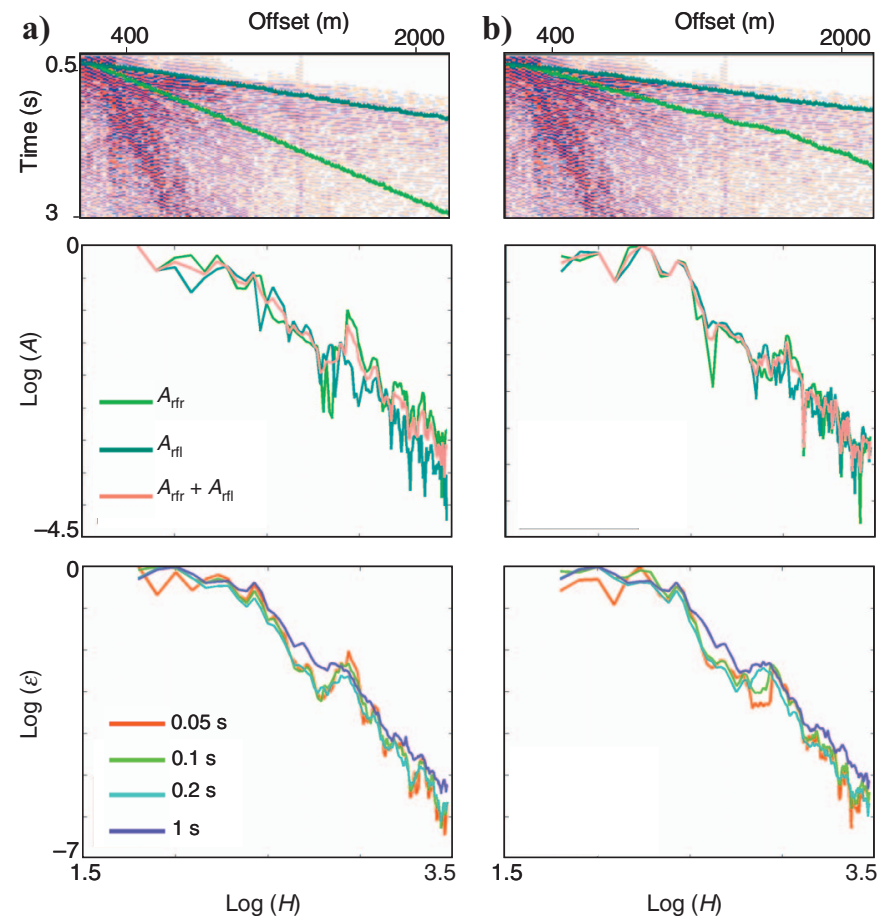

Figure 8. (Row 1) Refracted and reflected traveltime curves picked on traces from the real data and calculated separately for the (a) first, (b) second, and (c) third layers as well as (d) their total sum. (Row 2) Amplitude decays along the refracted and reflected traveltime curves and their sum. (Row 3 ) Corresponding energy decays for several values of window size $W_{t}$. See Figure 5.

Figure 9. Error curves obtained for the real data for unperturbed and perturbed depths (stars), velocity (plus signs), and both of them (circles) in the first layer, using new simulations. The filled circles show the minimum.
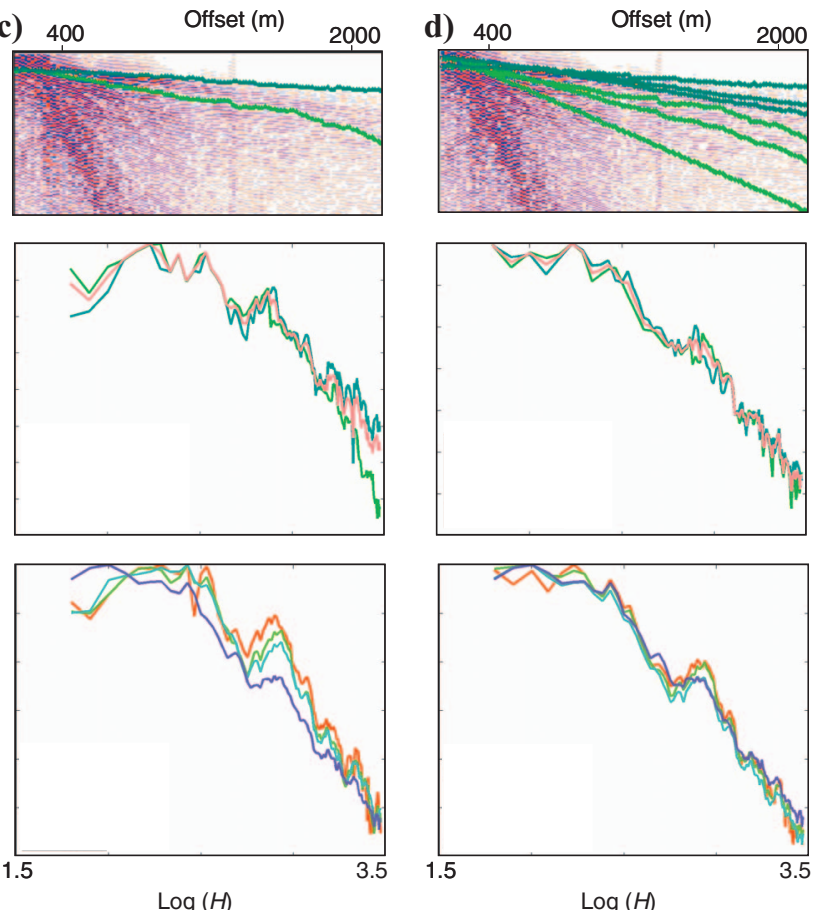

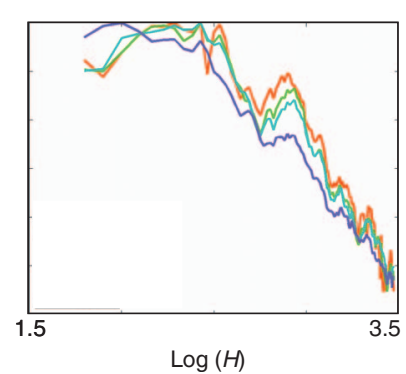

To further investigate the robustness of the picked refracted and reflected traveltimes, we did not repeat the finite-difference simulations for each perturbation because of computation costs. Instead, we reflected and refracted traveltimes while perturbing by $+2 \%$ up to $+10 \%$ separately or simultaneously the the first three layers, or all of them. The results are illustrated in Figure 10. Even if we change the picked traveltimes, we obtain the same estimated attenuation. This demonstrates the robustness of our method when inverting the $\mathbf{Q}$ of the first layer.

c) 400

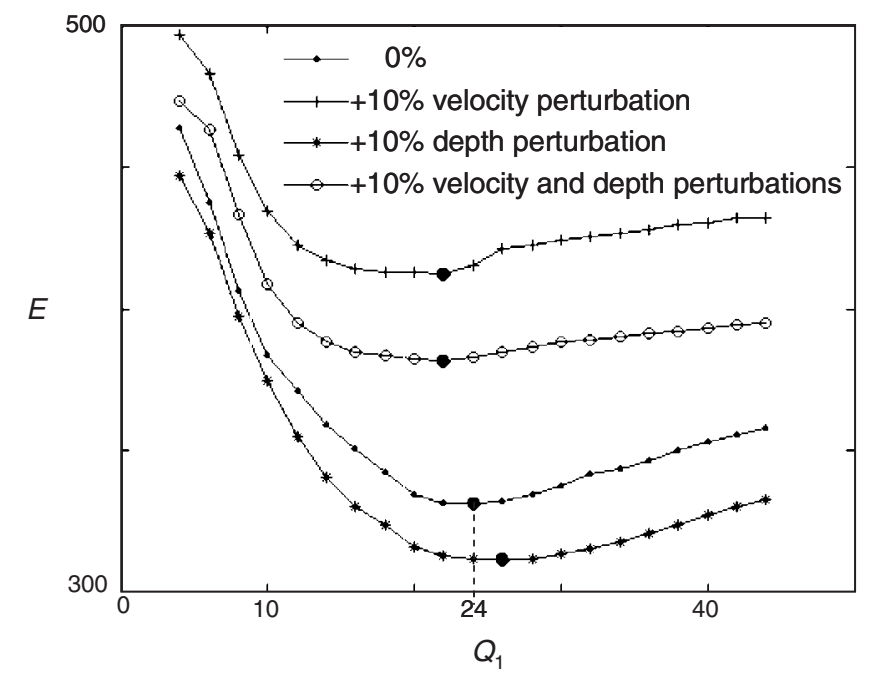


a)
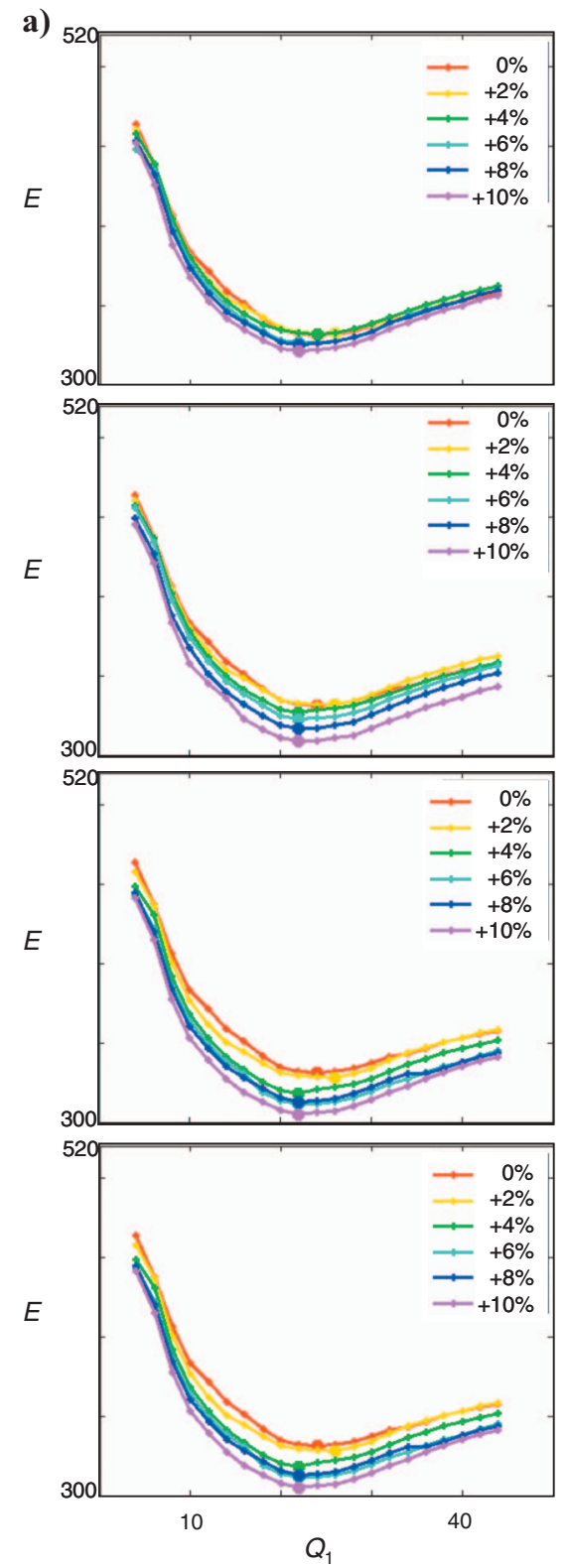

b)
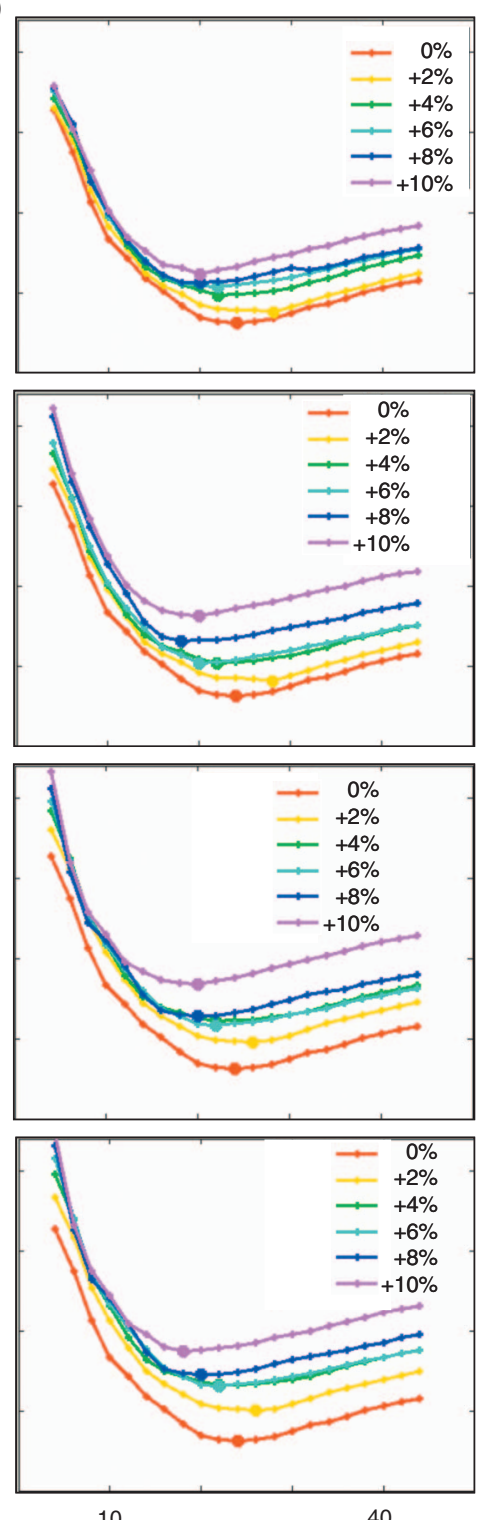

$Q_{1}$ c)
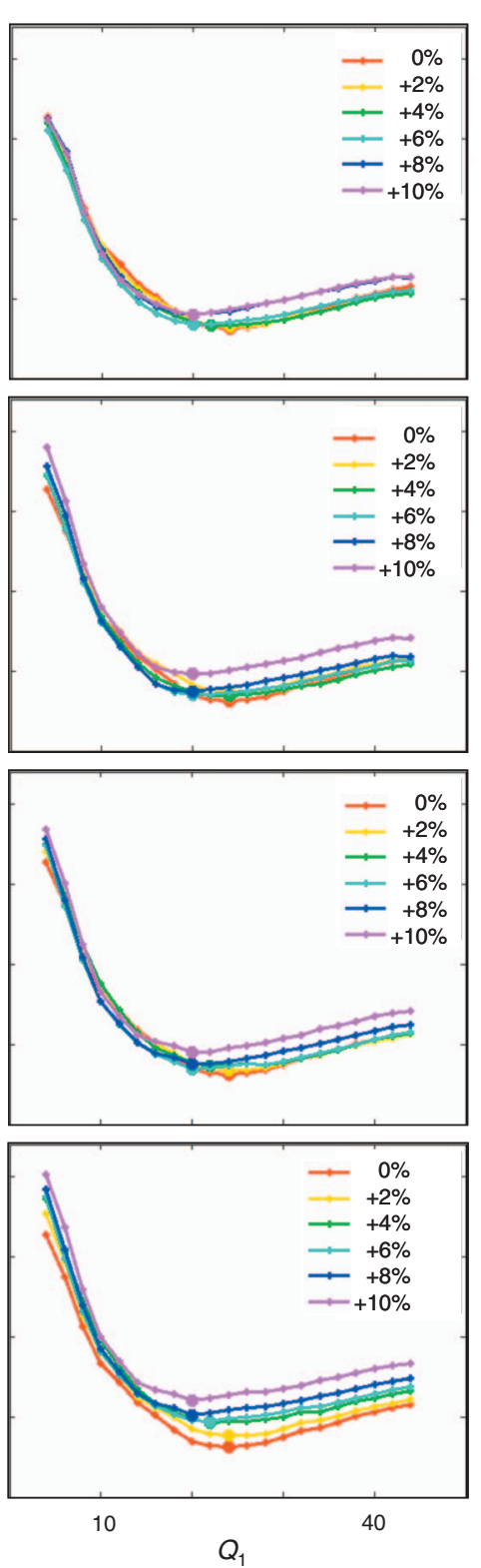

Figure 10. Error curves obtained for the real data for a range of (a) depth, (b) velocity, or (c) depth and velocity perturbations from $0 \%$ to $+10 \%$ without repeating the simulations. Per each column, we show (top to bottom) the results when perturbing only the first layer, the first two layers, the first three layers, or all of the layers, respectively. 

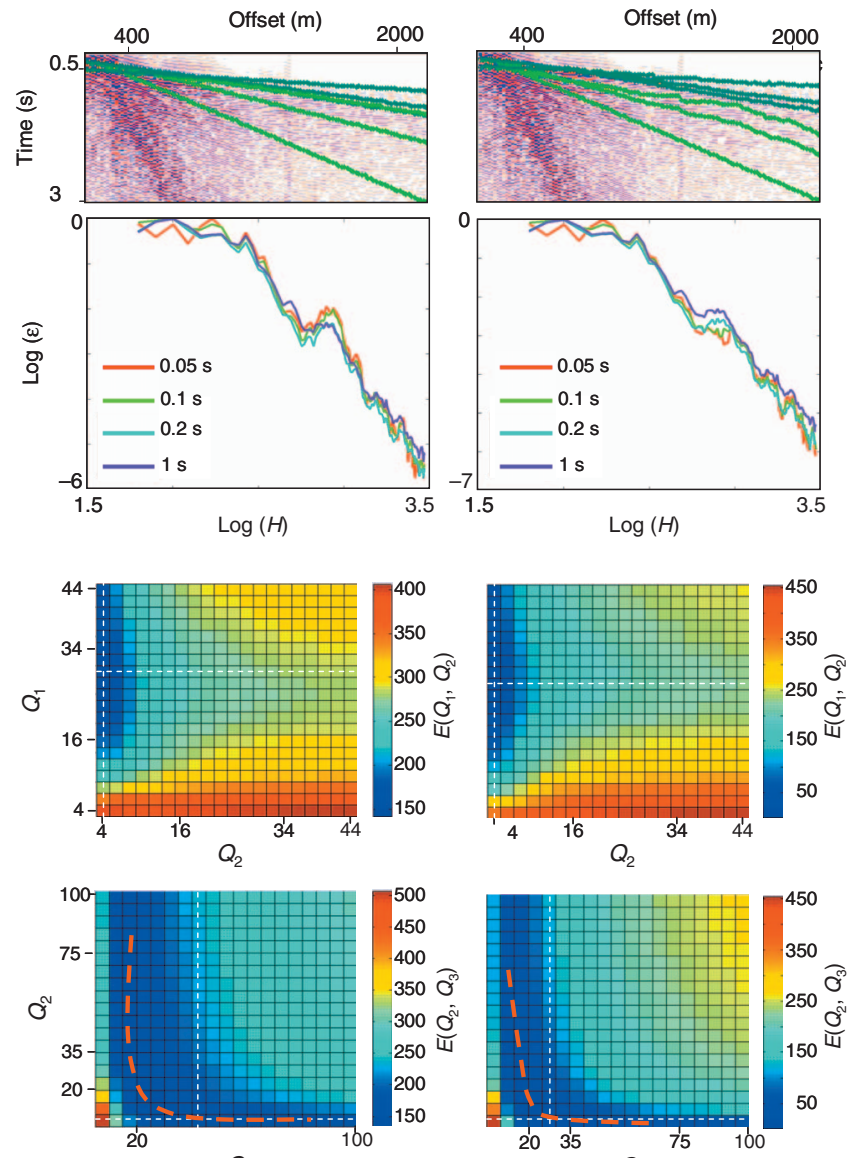

$Q_{3}$

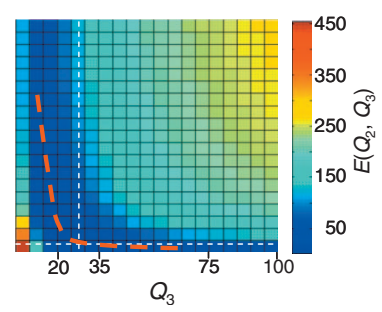

Figure 11. Traveltime curves (row 1) and their corresponding energy decay (row 2). The curves on the left were obtained without elevation corrections; those on the right, with corrections. Error images were calculated for the field data in both cases, (left) without and (right) with elevations corrections. The bottom panels were obtained for a fixed value of $Q_{1}=26$.

\section{Extension to multiple layers}

We want to extend our near-surface method to estimate the attenuation for multiple layers from the field data. Figure $3 \mathrm{~b}$ shows the source and receiver elevations for the real data set. We amended the traveltimes for elevation corrections in the observed seismogram but did not include topography nor elevation corrections in the finitedifference modeling.

The effects of elevation corrections on the picked traveltimes and their corresponding amplitude and energy decay are displayed in Figure 11, with no corrections shown on the left and corrections shown on the right. The energy values related to $W_{t}=0.1 \mathrm{~s}$ were inserted into the error criterion to estimate how well $Q_{1}, Q_{2}$, and $Q_{3}$ explain the data. The results show a reasonably smooth behavior of all images. Note the slightly enhanced resolution of the image obtained with elevation corrections, especially for the deeper layers. The value of $Q_{1}$ obtained at the minimum is 26 , slightly larger than the estimate when considering only the top layer. It agrees with typical regional values inferred from shear-wave $\mathbf{Q}$ measurements and the ratio between shear-wave and compressional-wave attenuation given by Mokhtar et al. (1988). However, we appear to obtain a value of four for $Q_{2}$, whereas we would expect a value $\geq Q_{1}$. One explanation could be that $Q_{2}$ is about 20, implying that $Q_{3}$ is about 20 as well, judging from the right lower panel along the red dashed line.

Figure 11 suggests that optimization of the attenuation parameters in the near surface should be feasible (see red dashed lines), either in a deterministic or a probabilistic sense. Layer stripping may be the way to go. First we can determine $Q_{1}$ by analyzing the behavior of $Q_{1}$ and $Q_{2}$, as in the top row of Figure 11. Then we can estimate $Q_{2}$ by considering $Q_{2}$ and $Q_{3}$ at the fixed estimated value of $Q_{1}$, as in the second row of Figure 11, and so on.

\section{CONCLUSIONS}

We have presented a method to estimate attenuation based on wave-propagation modeling and traveltime curves. The method was applied to a data set recorded in the Middle East. We used a viscoacoustic frequency-domain, finite-difference modeling code, and we started by estimating the attenuation of only the first layer. A sensitivity study indicated that the picked reflected and refracted traveltimes and the near-surface model from refraction statics provided sufficient robustness.

We included some of the complexity involved in the near surface. We incorporated elevation corrections in the reflection and refraction traveltimes used for picking amplitudes. The results showed a slightly enhanced resolution of the error image obtained with elevation corrections. The effect of geometric spreading and the source wavelet were taken into account. The wavelet should be carefully estimated. Finally, we extended the technique to multiple layers.

We applied the method to synthetic and field data and expect the estimated attenuation factors for real data to be reasonably accurate. The next step is to extend the method to the viscoelastic case as well as use a finite-difference code that includes topography.

\section{ACKNOWLEDGMENTS}

We would like to thank Shell International Exploration and Production BV as well as the South Rub Al Khali Company Ltd. (SRAK) and the Ministry of Petroleum and Mineral Resources of the Kingdom of Saudi Arabia for permission to use the seismic data.

\section{REFERENCES}

Alkhalifah, T., and C. Bagaini, 2006, Straight-rays redatuming: A fast and robust alternative to wave-equation-based datuming: Geophysics, 71, no. 3, U37-U46.

Alkhalifah, T., H. Innemee, and C. Benson, 2007, Straight ray datuming in 3D media - Fast and flexible: 69th EAGE Conference and Exhibition, Extended Abstracts, E037.

Ayres, A., and F. Theilen, 2001, Preliminary laboratory investigations into the attenuation of compressional and shear waves on near-surface marine sediments: Geophysical Prospecting, 49, 120-127.

Berryhill, J. R., 1979, Wave equation datuming: Geophysics, 44, 1329-1344. , 1984 , Wave equation datuming before stack (short note): Geophysics, 49, 2064-2066.

Blanch, J. O., A. Robertsson, and W. W. Symes, 1995, Modeling of a constant $Q$ : Methodology and algorithm for an efficient and optimally inexpensive viscoelastic technique: Geophysics, 60, 176-184.

Blanch, J. O., W. W. Symes, and R. J. Versteeg, 1998, A numerical study of linear viscoacoustic inversion, in R. G. Keys and D. J. Foster, eds., Comparison of seismic inversion methods on a single real data set: SEG, 13-44.

Bohm, G., F. Accaino, G. Rossi, and U. Tinivella, 2006, Tomographic joint inversion of first arrivals in a real case from Saudi Arabia: Geophysical Prospecting, 54, 721-730.

Booker, A. H., A. F. Linville, and C. B. Wason, 1976, Long wavelength static estimation: Geophysics, 41, 939-959.

Carcione, J. M., D. Kosloff, and R. Kosloff, 1988, Wave propagation simulation in a linear viscoacoustic medium: Geophysical Journal of the Royal 
Astronomical Society, 93, 393-407. Erratum: 1988, 95, 642

Christensen, R. M., 1982. Theory of viscoelasticity - An introduction, 2nd ed.: Academic Press Inc.

Cooke, D. A., and W. A. Schneider, 1983, Generalized linear inversion of reflection seismic data: Geophysics, 48, 665-676.

Coppens, F., 1985, First-arrival picking on common-offset trace collections for automatic estimation of static corrections: Geophysical Prospecting, 33, 1212-1231.

Cox, M., 1999, Static corrections for seismic reflection surveys: SEG.

Crase, E., A. Pica, M. Noble, J. McDonald, and A. Tarantola, 1990, Robust elastic nonlinear waveform inversion: Application to real data: Geophysics, 55, 527-538.

Dasgupta, R., and R. A. Clark, 1998, Estimation of $Q$ from surface seismic reflection data: Geophysics, 63,2120-2128.

El Yadari, N.., F. Ernst, and W. Mulder, 2007a, Near-surface attenuation estimation: 69th EAGE Conference and Exhibition, Extended Abstracts, E039.

2007b, Improvement of near-surface attenuation estimation: 77th Annual International Meeting, SEG, Expanded Abstracts, 1242-1246.

Engquist, B., and A. Majda, 1977, Absorbing boundary conditions for the numerical simulation of waves: Mathematics of Computation, 31, 629-651.

Ernst, F. E., 2007, Long-wavelength statics estimation from guided waves: 69th EAGE Conference and Exhibition, Extended Abstracts, E033.

Hauge, P. S., 1981, Measurements of attenuation from vertical seismic profiles: Geophysics, 46, 1548-1558.

Hicks, G. J., and R. G. Pratt, 2001, Reflection waveform inversion using local descent methods: Estimating attenuation and velocity over a gas-sand deposit: Geophysics, 66, 598-612.

Ley, R., R. Bridle, D. Amarasinghe, M. Al-Hamaili, M. Al-Ali, M. Zinger, and W. Rowe, 2003, Development of near surface models in Saudi Arabia for low relief structures and complex near-surface geology: 73rd Annual International Meeting, SEG, Expanded Abstracts, 1992-1995.

Malinowski, M., and S. Operto, 2006, Benefits of full-waveform modelling and inversion: A case study from the Polish Basin: 68th EAGE Conference and Exhibition, Extended Abstracts, P311.

2007, Multiparameter full-waveform inversion for velocity and attenuation - Refining the imaging of a sedimentary basin: 69th EAGE Conference and Exhibition, Extended Abstracts, P276.

Max, J., 1972, Méthodes et techniques de traitement de signal: Masson.

Minster, J. B., 1980, Anelasticity and attenuation, in A. Dziewonski and E. Boschi, eds., Proceedings of Enrico Fermi International School of Physics: Academic Press, 152-212.

Mokhtar, T. A., R. B. Herrmann, and D. R. Russell, 1988, Seismic velocity and $Q$ model for the shallow structure of the Arabian Shield from short-period Rayleigh waves: Geophysics, 53, 1379-1387.

Mulder, W. A., 2005, Rigorous redatuming: Geophysical Journal International, 161, 401-415.

Mulder, W. A., and R.-E. Plessix, 2004, A comparison between one-way and two-way wave-equation migration: Geophysics, 69, 1491-1504.

EA 2006, Full waveform tomography and nonlinear migration: 68th EAGE Conference and Exhibition, Extended Abstracts, A038.

Muller, G., 1983, Rheological properties and velocity dispersion of a medium with power-law dependence of Q on frequency: Geophysical Journal International, 54, 20-29.

O'Neil, M. E., and D. P. Hill, 1979, Causal absorption: Its effect on synthetic seismograms computed by the reflectivity method: Bulletin of the Seismological Society of America, 69, 17-25.
Operto, S., J. Virieux, J. X. Dessa, and G. Pascal, 2006, Crustal-scale seismic imaging from multifold ocean bottom seismometer data by frequency-domain full-waveform tomography: Application to the eastern Nankai Trough: Journal of Geophysical Research, 11, B09306.

Operto, S., S. Xu, and G. Lambaré, 2000, Can we quantitatively image complex structures with rays? Geophysics, 65, 1223-1238.

Pratt, R. G., 1999, Seismic waveform inversion in the frequency domain Part 1: Theory and verification in a physical scale model: Geophysics, 64, $888-901$.

Pratt, R. G., F. Hou, K. Bauer, and M. Weber, 2005, Waveform tomography images of velocity and inelastic attenuation from the Mallik 2002 crosshole seismic surveys: Geological Survey of Canada Bulletin, 585, 1-14.

Pratt, R. G., and R. M. Shipp, 1999, Seismic waveform inversion in the frequency domain - Part 2: Fault delineation in sediments using crosshole data: Geophysics, 64, 902-914.

Profeta, M., J. Moscoso, and M. Korembit, 1995, Minimum field static corrections: The Leading Edge, 89, 684-687.

Ravaut, C., S. Operto, L. Improta, J. Virieux, A. Herrero, and P. Del Aversana, 2004, Multiscale imaging of complex structures from multifold wide-aperture seismic data by frequency-domain full-waveform tomography: Application to a thrust belt: Geophysical Journal International, 159, 1032-1056.

Ribodetti, A., R.-E. Plessix, S. Operto, and J. M. Virieux, 2007, Viscoacoustic frequency-domain full-waveform inversion - Application to numerical VSP data: 69th EAGE Conference and Exhibition, Extended Abstracts, C09.

Ribodetti, A., and J. Virieux, 1998, Asymptotic theory for imaging the attenuation factor $Q$ : Geophysics, 63, 1767-1778.

Rickett, J., 2006, Integrated estimation of interval-attenuation profiles: Geophysics, 71, no. 4, A19-A23.

, 2007, Estimating attenuation and the relative information content of amplitude and phase spectra: Geophysics, 72, no. 1, R19-R27.

Robinson, D. K., and M. I. Al-Husseini, 1982, Technique for reflection prospecting in the Rub' Al-Khali: Geophysics, 47, 1135-1152.

Rogers, A., 1981, Determination of static corrections, in A. Fitch, ed., Developments in geophysical exploration methods: Applied Science Publishers, Ltd., 1-36.

Shtivelman, V., and A. Canning, 1988, Datum correction by wave-equation extrapolation: Geophysics, 53, 1311-1322.

Sirgue, L., and R. G. Pratt, 2004, Efficient waveform inversion and imaging: A strategy for selecting temporal frequencies: Geophysics, 69, 231-248.

Tarantola, A., 1984, Inversion of seismic reflection data in the acoustic approximation: Geophysics, 49, 1259-1266.

Toksöz, M., and D. Johnston, 1981, Seismic wave attenuation: SEG

Tonn, R., 1991, The determination of the seismic quality factor $Q$ from VSP data: A comparison of different computational methods: Geophysical Prospecting, 39, no. 1, 1-28.

Xia, J., R. D. Miller, and C. B. Park, 1999, Estimation of near-surface shearwave velocity by inversion of Rayleigh waves: Geophysics, 64, 691-700.

Xia, J., R. D. Miller, C. B. Park, and G. Tian, 2002, Determining $Q$ of nearsurface materials from Rayleigh waves: Journal of Applied Geophysics, $\mathbf{5 1}, 121-129$.

Zanzi, L., 1990, Inversion of refraction arrivals: A few problems: Geophysical Prospecting, 38, 339-364.

Zanzi, L., and A. Carlini, 1991, Refraction statics in the wavenumber domain: Geophysics, 56, 1661-1670. 